\title{
Can CK-MB be used as a marker in benign paroxysmal positional vertigo attack?
}

Mümtaz Taner Torun

Yusuf Yalçın

Özalkan Özkan

\begin{abstract}
Objectives: Benign paroxysmal positional vertigo (BPPV) is one of the main causes of vertigo. More than $90 \%$ of cases of positional vertigo and nystagmus can be associated with BPPV. There is no known specific laboratory test for BPPV. Because of the possible muscle activity in BPPV (especially result of nause, vomiting and increasing heart rate) we thought the CK-MB levels may change in this situation. The purpose of this study was to investigate the relation between serum CK-MB levels and BPPV and attacks. Materials and Methods: The medical records of 35 patients with BPPV presenting to our clinic between November 2014 and November 2015 were investigated retrospectively. Thirty-two control patients with no vertigo, muscular or cardiac disease and with measured CK-MB levels were randomized into a control group. Both groups' existing CK-MB levels were obtained from the patient records. CK-MB values measured before BPPV attack from 11 patients in the patient group were also recorded. Results: The patient and control groups were similar in terms of demographic parameters. CK-MB values at time of attack in the patient group ranged between 5.4 and 38.9 (mean: $19.67 \pm 6.81$ ), while control group values ranged between 12 and 27.9 (mean: $20.31 \pm 4.54$ ). CK-MB values in 11 patients in the patient group measured before attack ranged between 8.9 and 24 (mean: $17.02 \pm$ 4.97), and CK-MB values during attack ranged between 9 and 34 (mean: $19.74 \pm 7.64$ ). Conclusion: Although CK-MB elevation at time of attack was determined in patients with BPPV, this was not statistically significant.
\end{abstract}

Keywords: vertigo, nystagmus, pathologic, biomarkers, pharmacological, creatine kinas, MB form

University of Tuiuti in Paraná-Curitiba -PR-Brazil. Email: dr2735@mynet.com, dryusufyalcin@gmail.com,

ozalkanozkan@gmail.com

Institution: Bandırma State Hospital, Bandırma/Balıkesir, Turkey

Send correspondence to:

Mümtaz Taner Torun

Çanakkale Road 6th Km. Bandırma/Balıkesir, Turkey.81200-230. E-mail: dr2735@mynet.com

Paper submitted to the ITJ-EM (Editorial Manager System) on June 17, 2016;

and accepted on August 23, 2016. 


\section{INTRODUCTION}

The most commonly determined peripheral vestibular disease in patients presenting to health institutions with vertigo is benign paroxysmal positional vertigo (BPPV) ${ }^{1}$. BPPV is characterized by brief attacks that emerge with specific head positions and that resolve spontaneously. The annual prevalence is reported at $17 / 100,000$ in Japan and $64 / 100,000$ in the USA ${ }^{2,3}$. No prevalence for Turkey has been reported. It is twice as common in females as in males ${ }^{3,4}$.

BPPV results from abnormal mechanical stimulus and altered dynamics in the semicircular canals (SCC) in the middle ear following sudden movement of the head. Head rotation movements are perceived by the SCCs. Vertigo is thought to result from otoconia migrating from the utricle into the $\mathrm{SSC}^{4,5}$.

Creatine kinase $(\mathrm{CK})$ is a dimeric enzyme consisting of two subunits, each of $40 \mathrm{kD}$. It has three different isoenzymes, CK-MM (muscle type), CK-BB (brain type) and CK-MB (myocardial type). A fourth $64 \mathrm{kD}$ form with a different structure is located in the mitochondria and gives rise to $250 \mathrm{kD}$ oligomers known as macro CK II (MCK-II) when released into the circulation ${ }^{6}$. Although CK$\mathrm{MM}$ is dominant in both heart and skeletal muscle, more myocardium-specific CK-MB represents $10-20 \%$ of serum total CK activity, with CK-MB activity in skeletal muscle representing $2-5 \% \%^{7,8}$.

Rödöö and Hellberg investigated the CK-MB levels in children population and they suggested that CK-MB might be a diagnostic marker in benign paroxysmal vertigo $^{9}$. There are no laboratory tests used in the diagnosis of BPPV. The purpose of this study was to investigate the relation between serum CK-MB levels and BPPV and to assess whether this can be used as an attack marker.

\section{MATERIALS AND METHODS}

A retrospective case-control study designed. The medical files of 350 patients with vertigo presenting to our clinic in November 2014-November 2015 were investigated retrospectively. Seventy eight of them were BPPV and 35 of them had CK-MB assay. So 35 files with CK-MB values included into the study. Eighty one files investigated for control group. Thirty-two control patients that reported no vertigo, muscular or cardiac disease in their files and with measured CK-MB and troponin I levels were randomly selected and enrolled as the control group from 81 files. According to the patients' reports in their medical files, subjects aged under 18, pregnant women, subjects with high troponin-I values, with known muscle disease or epilepsy, using medications that affect muscle activity or with vestibular symptoms other than positional vertigo were excluded from both groups. The ages, gender and demographic datas were similar in both groups. Both groups' existing CK-MB values were recorded from the medical files. Additionally, CK-MB values measured before BPPV attack for 11 patients were also recorded from the medical files.

Other recorded biochemical parameters and blood pressures in the study group were within normal limits. In our clinic, we perform the maneuvers to suspicious positionel vertigo patients and keep their records on their medical files. Posterior BPPV is diagnosed on the basis of torsional nystagmus generally lasting less than $30 \mathrm{sec}$, counter-clockwise with the right ear beneath and clockwise with the left ear beneath, following a 10-15 sec latent period at the Dix-Hallpike maneuver, and on vertigo being concurrent with nystagmus. The supine roll test is the preferred maneuver to diagnose lateral canal BPPV. The supine roll test is performed by initially positioning the patient supine with the head in neutral position followed by quickly rotating the head $90^{\circ}$ to one side with the clinician observing the patient's eyes for nystagmus. After the nystagmus subsides (or if no nystagmus is elicited), the head is then returned to the straight faceup supine position. After any additional elicited nystagmus has subsided, the head is then quickly turned $90^{\circ}$ to the opposite side, and the eyes are once again observed for nystagmus. The nystagmus should be vertical and geotropic: rightward with the right ear beneath and leftward with lefte ar beneath. Other minor symptoms include tonus loss, pallor, nausea, vomiting, and muscle tension or spasm.

CK-MB activity in our hospital is measured using the immunoinhibition method with a Hitachi Modular p800 (Roche, Switzerland) chemistry analyzer. Study group results were accessed from the hospital laboratory data system. The 0-24 U/L is the reference range for CK-MB.

Statistical Analysis: Descriptive statistics were used to describe constant variables (mean, standard deviation, minimum, median, maximum). Normality of data distribution was investigated using the ShapiroWilk test. Comparison of two independent groups with normal distribution was performed using Student's t test. Variation between two matched groups without normal distribution was investigated using the Wilcoxon Signed Rank test. The chi square test was used to investigate relations between discrete. Statistical significance was set at p 0.05. Analyses were performed on MedCalc12.7.7. (MedCalc Software bvba, Ostend, Belgium; http://www. medcalc.org; 2013) statistical software).

\section{RESULTS}

Ages in the patient group ranged between 19 and 85 years (mean: $53.2 \pm 14.2$ ). Thirteen $(37.1 \%)$ patients were women and $22(62.9 \%)$ were men. Ages in the control group ranged between 18 and 87 years (mean: $47.7 \pm$ 19.6). Thirteen (40.6\%) were women and 19 (59.4\%) were men. There were no significant differences between the two groups in terms of gender. There was no statistically significant difference between the patient and control groups in terms of age, too (Student's t test $p=0.205$ ). Statistical data by age in the two groups are shown in the (Table 1). 
Table 1. Statistical values for group age distributions.

\begin{tabular}{lcc} 
& Patient group & Control group \\
\hline Patients & 35 & 32 \\
Mean & 53.20 & 47.78 \\
Median & 53.00 & 44.50 \\
Standard deviation & 14.256 & 19.638 \\
Minimum & 19 & 18 \\
Maximum & 85 & 87 \\
\hline
\end{tabular}

Patient group CK-MB values at time of attack were 5.4-38.9 (mean: $19.67 \pm 6.81$ ), while control group CKMB values were 12-27.9 (mean: $20.31 \pm 4.54$ ) (Graphic 1). No statistically significant difference was determined between the patient and control groups in terms of CKMB (Student's $t$ test $p=0.658$ ).

CK-MB values measured pre-attack in 11 patients in the patient group ranged between 8.9 and 24 (mean: $17.02 \pm 4.97$ ), while CK-MB values measured during attack ranged between 9 and 34 (mean: $19.74 \pm 7.64$ ) (Graphic 2). Pre-attack CK-MB values did not differ significantly from values measured during attack in the patient group (Wilcoxon Signed Rank test $p=0.223$ ).

\section{DISCUSSION}

Vertigo has a number of causes, the most common being BPPV, Meniere's syndrome and sudden hearing loss. Rare causes include labyrinthitis, vestibular schwannoma, delayed endolymphatic hydrops, Ramsey Hunt syndrome, otosyphilis, vestibular neuronitis, temporal bone fractures, cerebellar infarct, epilepsy and cervical vertigo ${ }^{10,11}$.

$B P P V$ is a generally idiopathic disease characterized by recurring vertigo attacks. Free movement of otoliths in the cupula or semicircular canals is widely implicated in the pathogenesis. Idiopathic BPPV is more common in the elderly and in women, and peaks in the $6^{\text {th }}$ decade ${ }^{4}$. One study reported a $0.5 \%$ level of BPPV attack in the $18-39$ age group, rising to $3.4 \%$ over the age of 60 . That study also reported a cumulative incidence of BPPV as high as $10 \%$ in the $80 \mathrm{~s}$, and that it is twice as common in women ${ }^{12}$. BPPV was more common in men in our study group, while the mean age was compatible with the literature. Vertigo may develop in association with natural age-related degeneration of the otolithic membrane over the age of 50 . While the known causes of BPPV include head trauma, migraine, ototoxicity, viral infections, Meniere's disease, long-term immobilization and ear operations, no cause can be identified in the majority of cases $^{5}$

$\mathrm{CK}$ is a basic muscle metabolism enzyme that catalyzes ATP-mediated creatine phosphorylation in a reversible manner ${ }^{13}$. CK-MB represents $20 \%$ of total myocardial CK activity ${ }^{14}$. It may be present at levels of less than $5 \%$ in prostate, spleen and skeletal muscle. CK-MB is released into the circulation from the affected muscle following acute myocardial infarction (AMI). Secretion begins approximately 2-4 $\mathrm{h}$ following AMI,

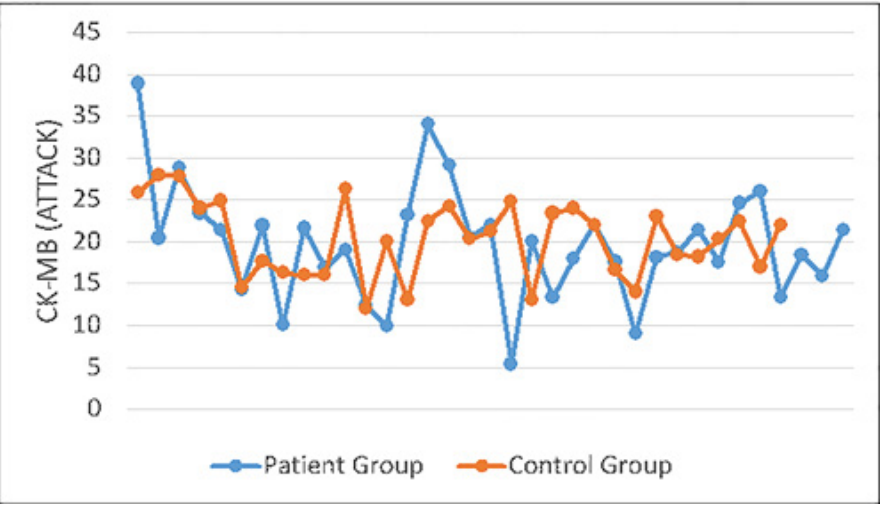

Graphic 1. CK-MB values in the control group and in the patient group at time of attack.

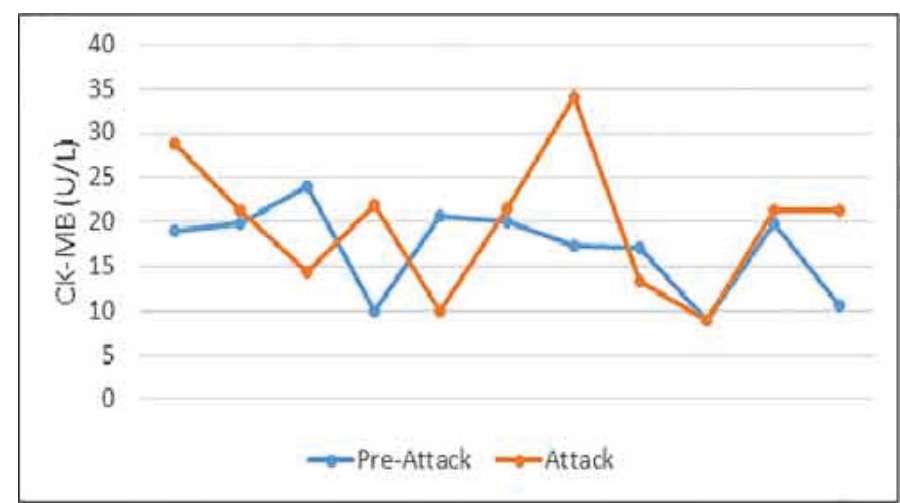

Graphic 2. Variation between time of attack and pre-attack CK-MB in the patient group.

peaks at $24 \mathrm{~h}$ and returns to normal in $36-72$ hours $^{13}$. CKMB measurement also assists in a non-invasive manner with the determination of reperfusion efficacy following thrombolytic therapy. CK-MB also increases in skeletal muscle injury. However, it is not as characteristic as in myocardial injury ${ }^{13}$. CK-MB assay can be performed by measuring activity or mass. CK-MB activity is measured using immunoinhibition technology ${ }^{15}$.

Simultaneous vertigo with torsional nystagmus using the Dix-Hallpike maneuver is regarded as the most significant criterion in the diagnosis of posterior canal BPPV. However, this maneuver may be insufficient in horizontal, anterior and mixed canal-type BPPV, and additional tests, such as the Roll test and Barbeque and Semont maneuvers, may be required in other forms of BPPV. Nausea, vomiting and neurovegetative symptoms experienced during BPPV can increase muscle activity and heart rate and it may change CK-MB levels. In these cases it's important to recognize the CK-MB levels might change in the hearth diseases, so troponın I rates must be controlled.

Various laboratory markers have been investigated in recent years for the purpose of overcoming these deficiencies, for differential diagnosis and to predict time of attack. A biomarker that can be used in all types of BPPV can provide a faster and proper diagnose. Özbay et al. investigated neutrophil-lymphocyte ratios in patients with peripheral vertigo, and reported significantly higher 
results compared to the healthy population ${ }^{11}$. Parham et al. reported significant elevation in BPPV of a proteinous substance known as otolin-1 released from otoconia and vestibular and cochlear cells ${ }^{16}$. One study reporting that low serum vitamin $\mathrm{D}$ and osteoporosis may also be a risk factor in BPPV determined a higher body mass index in patients with BPPV ${ }^{17}$. Another similar study reported a higher incidence of idiopathic BPPV in women with postmenopausal osteopenia or osteoporosis ${ }^{18}$. In their study of 55 patients, Yuan et al. investigated serum albumin and globulin levels and determined significantly higher y-globulin levels at time of BPPV attack compared to control subjects ${ }^{19}$. In another study, they investigated uric acid levels in patients with BPPV, but determined no significant variation ${ }^{20}$.

Akıncı et al. investigated fibrinogen, D-dimer and C-reactive protein as markers that might be used in the differential diagnosis of central and peripheral vertigo, but identified no significant variations ${ }^{21}$. As these studies show, the subject is still current and the search for markers is ongoing. Further studies concerning markers with significantly high or low levels are now needed.

Our study was therefore planned on the basis that an increase might be observed in serum CK-MB levels. The patients who had normal troponin-I values were evaluated for our study. Rödöö and Hellberg observed an increase in CK-MB levels in childhood benign paroxysmal vertigo and suggested that this might be associated with benign paroxysmal vertigo muscle activity and that $\mathrm{CM}-\mathrm{MB}$ might be a diagnostic marker in benign paroxysmal vertigo . Although CK-MB levels in our study were higher in some cases at time of attack than before attack, the difference was not statistically significant. The main reason for this may be the low patient number in this study. In addition, the absence of any time-specific standardization in the evaluation of CK-MB levels, particularly pre-attack, (CKMB levels have been assessed 2 months pre-attack in some studies and 5 months pre-attack in others) and the broad age scale are other potential causes. Additionally, the other diseases that may cause the alteration of CK-MB levels that doesn't report in the medical files is a handicap of the study.

\section{CONCLUSION}

Although CK-MB elevation was determined at time of attack in patients with BPPV in this study, this was not statistically significant. CK-MB may be developed as a marker at time of attack for BPPV as a result of prospective investigation in larger series in future studies.

\section{REFERENCES}

1. Anagnostou E, Kouzi I, Spengos K. Diagnosis and Treatment of Anterior-Canal Benign Paroxysmal Positional Vertigo: A Systematic Review. J Clin Neurol. 2015;11(3):262-7.
2. Bhattacharyya N, Baugh RF, Orvidas L, Barrs D, Bronston LJ, Cass $S$, et al. Clinical practice guideline: benign paroxysmal positional vertigo. Otolaryngol Head Neck Surg. 2008;139(5 Suppl 4):S47-81.

3. Froehling DA, Silverstein MD, Mohr DN, Beatty CW, Offord KP, Ballard DJ. Benign positional vertigo: incidence and prognosis in a population-based study in Olmsted County, Minnesota. Mayo ClinProc 1991;66(6):596-601.

4. Lee SH, KimJS. Benign Paroxysmal Positional Vertigo. J Clin Neurol. 2010;6(2):51-63.

5. Hancı D, Altun H. Prevalence of benign paroxysmal positional vertigo among motocross racers: a case-control study. Kulak Burun Bogaz Ihtis Derg. 2015;25(5):279-83.

6. Henry JB. Clinical diagnosis and management by laboratory methods. 20th ed. W.B Saunders Comp; 2001.

7. Burtis CA, Ashwood ER, Bruns DE. Tietz Textbook of clinical chemistry and molecular diagnostics 4th ed. W.B Saunders Comp; 2005.

8. Etem AA, Etem EÖ, Yıldırmak ST, Ulutaş GŞ, Ünal S. Comparison of CK-MB Mass and Activity Measurement Methods in Acute Myocardium Infarction and Healthy Groups. Journal of Turkish Clinical Biochemistry 2011;9(2): 53-9.

9. Rödöö P, Hellberg D. Creatine kinase MB (CK-MB) in benign paroxysmal vertigo of childhood: a new diagnostic marker. J Pediatr 2005; 146(4):548-51.

10. Orji F. The influence of psychological factors in Meniere's disease. Ann Med Health Sci Res. 2014;4:3-7.

11. Ozbay I, Kahraman C, Balıkcı HH, Kucur C, Kahraman NK, Ozkaya DP, et al. Neutrophil-to-lymphocyte ratio in patients with peripheral vertigo. A prospective controlled clinical study. Am J Otolaryngol. 2014;35(6):699-702

12. Von Brevern $M$, Radtke $A$, Lezius $F$, Feldmann $M$, Ziese $T$, Lempert T,et al (2007) Epidemiology of benign paroxysmal positional vertigo: a population based study. J Neurol Neurosurg Psychiatry.2007;78:710-15.

13. Newby LK, Ohman EM, Christenson RH. The role of the troponins and other markers of myocardial nekrosis in risk stratification. Topol EJ. Acute Coronary Syndromes. Second ed. New York: Marcel Dekker; 2001. p. 329-72.

14. Urdal P, Urdal K, Stromme JH. Cytoplasmic creatine kinase isoenzymes quantitated in tissue specimens obtained at surgery. Clin Chem. 1983;29:310-3.

15.Strom S, Bendz R. Serum creatine kinase (CK) activity after $\mathrm{M}$-subunit inhibition in patients with atypical CK isoenzymes-a comparison to findings in acute myocardial infarction. Clin Chem Acta. 1983;132:73-81.

16.Parham K, Sacks D, Bixby C, Fall P. Inner ear protein as a biomarker in circulation? Otolaryngol Head Neck Surg. 2014;151(6):1038-40.

17. Jeong SH, Kim JS, Shin JW, Kim S, Lee H, Lee AY, et al. Decreased serum vitamin $\mathrm{D}$ in idiopathic benign paroxysmal positional vertigo. J Neurol 2013;260:832-38.

18.Parham K, Leonard G, Feinn RS, Lafreniere D, Kenny AM. Prospective clinical investigation of the relationship between idiopathic benign paroxysmal positional vertigo and bone turnover: a pilot study. Laryngoscope 2013;123(11):2834-9.

19. Yuan $\mathrm{H}$, Song $\mathrm{Y}$, Zhang $D$. Clinical observation of the $\mathrm{y}$-globulin levels when benign paroxysmal positional vertigo is attacking. Lin Chung Er Bi Yan Hou Tou Jing Wai Ke Za Zhi. 2015;29(10):931-3.

20. Yuan J, Chen Y, Chen Y, Niu S, Li S, Dong Q, Hu W. Relationship between serum level of uric acid and benign paroxysmal positional vertigo. Zhonghua Yi XueZaZhi. 2015;95(5):344-8.

21.Akinci E, Aygencel G, Keles A, Demircan A, Bildik F. Role of C-reactive protein, D-dimer, and fibrinogen levels in the differential diagnosis of central and peripheral vertigo. Adv Ther. 2007;24(5):1068-77. 